\title{
Caracterización fisicoquímica de suelos salinos agrícolas, en la localidad de Chicavasco, estado de Hidalgo, México
}

\section{Physicochemical characterization of saline agricultural soils in the town of Chicavasco, Hidalgo State, Mexico}

DOI: $10.46932 / \mathrm{sfjdv} 3 \mathrm{n} 1-026$

Received in: Dec 30st, 2021

Accepted in: Jan 1th, 2022

\section{Cristián Raziel Delgado-González}

estudiante del Doctorado en Ciencias Agropecuarias en el Instituto en Ciencias Agropecuarias (ICAp) de la Universidad Autónoma del Estado de Hidalgo (UAEH), Tulancingo, Hidalgo

E-mail:mislas@uaeh.edu.mx

\section{Rodrigo Rodríguez-Laguna}

profesor investigador de la UAEH, Tulancingo

E-mail: Hidalgo mislas@uaeh.edu.mx

Juan Capulín-Grande

profesor investigador de la UAEH, Tulancingo

E-mail: Hidalgo mislas@uaeh.edu.mx

Alfredo Madariaga-Navarrete

profesor investigador de la UAEH, Tulancingo

E-mail: Hidalgo mislas@uaeh.edu.mx

Margarita Islas-Pelcastre

profesora investigadora de la UAEH, Tulancingo, Hidalgo

E-mail: mislas@uaeh.edu.mx

\section{RESUMEN}

Los suelos agrícolas salinos tienen altas concentraciones de sales solubles (cloruro de sodio), que afectan el crecimiento de cultivos, al disminuir la asimilación de nutrientes y la actividad microbiana. Las plantas pueden ser tolerantes a la salinidad del suelo, pero dependen de algunas propiedades como textura, conductividad eléctrica, $\mathrm{pH}$, entre otros. Este trabajo evaluó un suelo agrícola erosionado por salinidad, mediante parámetros físicos y químicos, para proponer un proceso biológico de recuperación. Los resultados mostraron alto contenido de sodio $(2750 \mathrm{ppm}), \mathrm{pH}$ de 8.2 , conductividad eléctrica de $8.5 \mathrm{dS} / \mathrm{m}$ y 19.89 de sodio intercambiable (ESP).Por esta razón, se puede clasificar como un suelo salino-sódico, además los altos valores de $\mathrm{pH}$ y conductividad eléctrica infieren baja disponibilidad de algunos micronutrientes, dificultando la absorción de estos a los cultivos. Las características fisicoquímicas del suelo refieren un suelo con baja fertilidad y poca probabilidad para el desarrollo vegetal.

Palabras clave: Salinidad, suelos, caracterización, fisicoquímicas, biodisponibilidad.

\section{ABSTRACT}

Saline agricultural soils have high concentrations of soluble salts (sodium chloride), which affect crop growth by reducing nutrient assimilation and microbial activity. Plants can be tolerant to soil salinity, but 
they depend on some properties such as texture, electrical conductivity, $\mathrm{pH}$, among others. This work evaluated an agricultural soil eroded by salinity, using physical and chemical parameters, to propose a biological recovery process. The results showed high sodium content $(2750 \mathrm{ppm}), \mathrm{pH}$ of 8.2 , electrical conductivity of $8.5 \mathrm{dS} / \mathrm{m}$ and 19.89 exchangeable sodium (ESP), which can be classified as a saline-sodic soil, and the high $\mathrm{pH}$ and electrical conductivity values infer low availability of some micronutrients, making their absorption by crops difficult. The physicochemical characteristics of the soil indicate a soil with low fertility and low probability for plant development.

Keywords: Salinity, soils, characterization, physicochemical, bioavailability.

\section{INTRODUCCIÓN}

La salinización es un ejemplo de la degradación del suelo agrícola. La causa de los procesos en la salinización puede ser multifactorial, existen de manera natural en las zonas áridas y semiáridas del planeta, se caracterizan por escasas precipitaciones y elevadas tasas de evaporación. Otro factor es el uso de agua de riego con alto contenido de sales, prácticas inapropiadas de cultivos, el uso excesivo de químicos, entre otros.

Lopez-Sanchez et al. (2018), refiere que "alrededor del 7 \% de las tierras cultivables en el mundo están afectadas por sales y para los próximos años se estima su incremento en más de un $20 \%$, por lo cual se considera la salinidad uno de los problemas más importantes para la agricultura a nivel internacional". Se estima que más del $40 \%$ de los suelos de uso agrícola a nivel mundial son sódicos y salinos, en consecuencia, las prácticas agrícolas en estos sitios son muy bajas. Incluso el rendimiento real producido a partir de suelos salinos promedia un 50\% menos que el rendimiento generado a partir de suelos normales. Por supuesto estos suelos tienen muy poca materia orgánica y biodiversidad (Rengasamy, 2002, citado por, Sameen et al., 2016).

El efecto negativo de la salinidad en los suelos agrícolas que impacta en la estructura del suelo y en el crecimiento de la planta es el potencial osmótico (Ahmad y Sharma, 2008; Sameen et al., 2016). La producción de materia orgánica, la transpiración de las plantas y el rendimiento en la biomasa se reducen significativamente; aunque esto depende directamente de las especies de la planta y el manejo del riego. Otro aspecto negativo significativo es la evapotranspiración, la resistencia estomática y el potencial hídrico (Katerji et al., 2000; Sameen et al., 2016).

La salinidad origina cambios en las propiedades físicas y químicas del suelo, por tener baja biodiversidad, deficiencias de nutrientes y alta toxicidad. Presentan una considerable concentración de iones boro, carbonato y aluminio. El porcentaje de los iones de sodio intercambiables aumentan con un aumento de la salinidad (Tedeschi y Dell'Aquilla, 2005), poseen altas concentraciones de iones de potasio, iones de cloruro y sulfato. Por otro lado, el aumento de la salinidad del suelo provoca una disminución en la concentración de iones de magnesio e iones de calcio en el suelo. Otras propiedades que se alteran por 
la salinidad es la baja estabilidad estructural, tasa de infiltración y capacidad de retención de agua (Hashem et al., 2014; Sarwar et al., 2017).

En suelos salinos que se debe evaluar composición de este biomaterial, dado que esta se le considera como una mezcla de materia mineral, orgánica agua y aire. Las proporciones de estos componentes varían de tiempo en tiempo y de lugar a lugar. En general está constituido por tres fases: fase sólida, fase líquida y fase gaseosa.

Determinar las propiedades del suelo salino como la textura, indica la composición granulométrica cuantitativa del suelo, que representa los valores de las tres fracciones principales (arcilla, limo y arena). En lo relativo a la estructura, es necesario conocer la forma de agregación que tienen los elementos minerales y orgánicos del suelo.

Asimismo, las propiedades químicas del suelo que se ven afectadas negativamente por la salinidad es el aumento de las concentraciones de iones de metales pesados y cadmio. Los iones de sodio también aumentan su concentración, seguido por los cloruros, los iones sulfato y bicarbonato (Cao et al., 2012). Los resultados de la salinidad retribuyen en la degradación de la materia orgánica, la reducción en la biomasa microbiana y la actividad microbiana, que a su vez cambia los flujos de dióxido de carbono (Wong et al., 2010).

La concentración de la salinidad del suelo se expresa comúnmente por la conductividad eléctrica y utilizando este parámetro, los suelos se pueden clasificar en tres grupos de acuerdo a (Chhabra, 2017; Isbell, 2016; Qadir et al., 2000).

- Suelos salinos: Conductividad eléctrica (CE) mayor a $4 \mathrm{dS} / \mathrm{m}$, porcentaje de adsorción de sodio mayor que 13 y porcentaje de sodio intercambiable por debajo de 15 .

- Suelos sódicos: CE por debajo de 4 dS/m, adsorción por debajo de 13 y sodio intercambiable por debajo de 15.

- Suelos salinos - sódicos: CE mayor a 4 dS/m, adsorción mayor a 13 y sodio intercambiable mayor a 15.

Finalmente, el $\mathrm{pH}$ en el suelo es una propiedad química que determina el comportamiento y evolución de nutrientes y contaminantes en los diversos perfiles del suelo, en la vegetación y microbiota (Raina et al., 2009). El pH del suelo mide la concentración de iones $\mathrm{H}^{+}$en la solución del suelo, está determinando por la composición de la solución y de sus componentes más abundantes; como cationes básicos: $\mathrm{Ca}^{2+}, \mathrm{Mg}^{2+}, \mathrm{K}^{+}$y Na${ }^{+}$y cationes ácidos: $\mathrm{H}^{+}, \mathrm{Al}^{+3}, \mathrm{Fe}^{+3}, \mathrm{NH}_{4}{ }^{+}$(Ortíz y Ortíz, 1988).

Se han reportado varias investigaciones para descubrir métodos mediante los cuales se puedan mejorar los suelos salinos para garantizar la seguridad alimentaria. Por ejemplo, Sameen et al. (2016), refiere que las propiedades físicas y químicas de los suelos salinos mejoran con el crecimiento de plantas 
tolerantes a la sal. En este sentido encontramos que la fitorremediación es una técnica biológica que utiliza las plantas para mejorar los suelos deteriorados. Existen plantas que pueden tolerar un alto contenido de sal y que se utilizan para la remediación de los suelos salinos. En aspectos fisicoquímicos, las plantas adecuadas que pueden mejorar los niveles de calcio y disminuir los niveles de sodio en el suelo (Gupta \& Goyal, 2017).

Se ha documentado que hay variedades de plantas que mejoran la absorción de agua en el suelo, la conductividad hidráulica saturada del suelo, la estabilidad estructural, la densidad aparente del suelo y la porosidad del mismo. En consecuencia, el aumento de agua disponible se asocia con el contenido de materia orgánica y la actividad microbiana (Sameen et al., 2016).

Nouri et al. (2017), documentan el potencial de las plantas y los microorganismos para la eliminación de xenobioticos en la remediación del suelo. Destacan que en la rizosfera hay procesos que inducen directamente por la actividad en las raíces de la planta estimulando la secreción de varios ácidos orgánicos que ayudada por los agentes quelantes de la planta, origina cambios de $\mathrm{pH}$ y reacciones redox, que incide en las propiedades físicas y químicas del suelo salino.

Esta revisión se centra en las características físicas y químicas de los suelos salinos, con la finalidad de identificar las formas de eliminar la sal de los suelos y con ello el aumento de la fertilidad del suelo.

\section{DESCRIPCIÓN DEL MÉTODO}

\subsection{LOCALIZACIÓN DEL ÁREA DE ESTUDIO}

El suelo agrícola se localiza en el municipio de Actopan, Hidalgo, en la región de Chicavasco, en

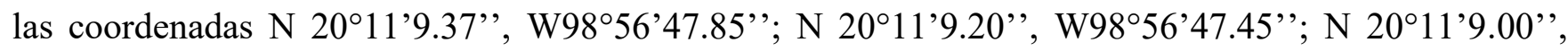
W98 56'48.10', y N 20¹1'8.80', W98 56'47.65' '.Registra clima semiseco templado, con temperatura media anual entre 16 y $20{ }^{\circ} \mathrm{C}$, precipitación anual media entre 400 - $560 \mathrm{~mm}$ (Servicio Meteorológico Nacional, 2018).

El muestreo, preparación y conservación de la muestra de suelo se realizó conforme a la NOM021-SEMARNAT-2000. También, esta norma establece los métodos analíticos de las propiedades físicas y químicas en los suelos experimentales se llevaron a cabo, donde se establece las especificaciones, de la fertilidad, salinidad y clasificación de suelos, estudio, muestreo y análisis

\subsection{DETERMINACIONES FISICOQUÍMICAS DEL SUELO}

Las determinaciones de propiedades físicas fueron: humedad por gravimetría (AS-05); textura por procedimiento de Bouyoucos (AS-09) y densidad real (AS-04).

En relación a las propiedades químicas: pH (AS-02, AS-23 y AS-24); conductividad eléctrica en el extracto de saturación (AS-18); materia orgánica (método Walkey y Black); nitrógeno total (AS-25); 
capacidad de intercambio catiónico y bases intercambiables (kit de análisis de suelo SMART 3 de LaMotte que refiere el método Mehlich, 1984); Calcio y magnesio (Método EDTA de Schwarzenbach); cloruros (método de lectura directa en el kit de análisis de suelo SMART 3 de LaMotte); cobre (método del dietilditiocarbamato); Hierro (método del bipiridil); manganeso (método del peryodato); fosfatos (método de la reducción de ácido ascórbico); potasio (método del tetrafenilboron); sodio (sodiómetro); PSI (NOM021, 2002) por ultimo sulfatos y zinc (kit de análisis de suelo SMART 3 de LaMotte que refiere el método Mehlich, 1984).

\section{RESULTADOS Y DISCUSIONES}

Las propiedades físicas del suelo experimental indican que la textura es franco arenoso, que se caracteriza por su porcentaje de arena, arcilla y limo (Tabla 1) (Vasques et al., 2014); la humedad oscila en el $14 \%$ lo que representan un nivel adecuado considerado lo que establecen Martínez-González y colaboradores (2010) para suelos con cobertura vegetal arbustiva o arbórea en la época de lluvias. Este valor pudo verse influenciado por la toma de muestra inmediata a la época de lluvias. Por último, la densidad real $\left(1.3 \mathrm{~g} / \mathrm{cm}^{3}\right)$, se encuentra dentro de los intervalos para suelos franco-arenosos (NOM021,2002).

Respecto a los parámetros químicos (Tabla 1) se reportan valores relacionados a suelos salinos. Por ejemplo, el pH promedio 8.2 que lo refiere a intervalos en suelos moderadamente alcalinos a fuertemente alcalinos. Éste parámetro es un indicador del comportamiento en la disponibilidad y distribución de los micro y algunos macro nutrientes. Además, hay una relación entre el contenido de materia orgánica, conductividad eléctrica y la concentración de sales solubles como el cloruro de sodio (Read et al., 2016).

Ahora bien, la cantidad de materia orgánica se considera moderada para suelos no volcánicos (2.5 \%) que coincide para suelos medianamente alcalinos. Zhalnina et al. (2015), menciona que bajos valores de materia orgánica pueden modificar la disponibilidad de algunos nutrientes, es un factor que afecta la diversidad microbiológica y por lo tanto a la diversidad de plantas en los ecosistemas. De igual forma esté autores infieren que la baja disponibilidad de materia orgánica tiene incidencia en la presencia de organismos fijadores de nitrógeno y por tanto explica la baja disponibilidad de Nitrógeno en el suelo de estudio (Tabla 1).

Por otro lado, la conductividad eléctrica (CE) está estrechamente relacionada con el pH y la cantidad de sales en el medio. La CE fue de $8.5 \mathrm{dS} / \mathrm{m}$, este valor confirma la salinidad del suelo y es un indicador de la cantidad y composición de sales solubles presentes, lo cual podría modificar la capacidad de intercambio de algunos nutrientes, específicamente potasio, calcio y magnesio (Behera y Shukla, 2014). También podría reflejar la cantidad excesiva de cloruros y de sodio presentes en el suelo (Tabla 1) 
que se relaciona directamente con los valores de $\mathrm{CE}$ y $\mathrm{pH}$. Al existir altas concentraciones de sales, específicamente $\mathrm{NaCl}$, el pH se incrementa a la par de los valores de $\mathrm{CE}$, modificando con ello una propiedad intrínseca del sodio que lo puede convertir en un elemento tóxico para las plantas, expresado como porcentaje intercambiable de sodio (PSI) que en este estudio fue de $19.89 \%$. Si el PSI es mayor a 15, la CE de $8.5 \mathrm{dS} / \mathrm{m}$, la adsorción de sodio mayor a 13, y un valor alto de $\mathrm{pH}$ (8.2), el suelo en estudio puede clasificarse como suelo salino - sódico (Chhabra, 2017; De Sutter et al., 2015; Huang et al., 2014; Isbell, 2016; Qadir et al., 2000), que podría dificultar el crecimiento vegetal y modificar la estructura e interacciones en la rizósfera (Zhalnina et al., 2015).

En relación a los micro elementos, se encontró que el Cobre $(\mathrm{Cu})$ se fija químicamente con facilidad en suelos alcalinos con valores de $\mathrm{pH}$ altos disminuyendo su disponibilidad. Los resultados presentados para el cobre están en límites bajos de este micronutriente (NOM-021). Duplay y colaboradores (2014), reportan una correlación positiva entre la biodisponibilidad del $\mathrm{Cu}$, la cantidad de materia orgánica y el pH en el suelo; donde se registraron valores más bajos de $\mathrm{Cu}$ (10 ppm) en suelos con $\mathrm{pH}$ por arriba de 8 , en contraste con suelos con un $\mathrm{pH}$ de 7 y valores alrededor de $20 \mathrm{ppm}$ de $\mathrm{Cu}$. De igual manera el Hierro $(\mathrm{Fe})$, a pesar de ser uno de los micronutrientes más abundantes, es el elemento que se encuentra en menor disponibilidad debido a los valores de $\mathrm{pH}$. Bravo et al. (2017), reportan valores de Hierro en suelos alcalinos ( $\mathrm{pH}>8)$ alrededor de 10 ppm, que son mucho más bajos que la media a nivel mundial de 40 ppm de Fe. Estos se comparan con los valores excesivamente bajos de Fe encontrados en el suelo alcalino estudiado con un pH por arriba de 8 (Tabla 1).

El Manganeso (Mn), se comporta de una manera similar al Fe, de acuerdo a Redžić et al. (2014), en suelos alcalinos y neutros con $\mathrm{pH}>7$ reportan que la biodisponibilidad de Manganeso disminuyó a 9 ppm en comparación con suelos de pH por debajo de 7, que fue de $15 \mathrm{ppm}$ de $\mathrm{Mn}$, existiendo una correlación negativa entre el aumento de $\mathrm{pH}$ y la biodisponibilidad del elemento, siendo semejante a los resultados mostrados en la tabla 1, ya que el pH del suelo es mayor a 8 la disponibilidad disminuye aún más. Con respecto al Zinc (Zn), su valor esta en los límites (0.65 ppm) marginales de acuerdo a la NOM021 y se relacionan a los valores de pH. Bravo et al. (2017), presentan que el Zn está en mayor disponibilidad en suelos ácidos a $\mathrm{pH}<7$ con valores cercanos a $7.8 \mathrm{ppm}$ mientras que suelos con $\mathrm{pH}>8$ los valores fueron por inferiores a $3.2 \mathrm{ppm}$ de $\mathrm{Zn}$.

El Fósforo (P) es un caso particular, ya que encuentra su óptimo en suelos con $\mathrm{pH}$ alrededor de 7 , con valores entre 2.3 a $5.1 \mathrm{Cmol}^{(+)} \mathrm{kg}^{-1}$ (Brooker et al., 2018). Comparado al suelo en estudio que presenta un valor de $\mathrm{pH}$ mayor a 8 , la disponibilidad registra un valor marginal/bajo y en consecuencia podría condicionar su absorción en las raíces de las plantas. Finalmente, la disponibilidad de Calcio (Ca) y Magnesio (Mg), están en intervalos altos, ya que estos elementos tienen un óptimo en ambientes alcalinos. La concentración normal de Ca se reporta entre los 2 y $20 \mathrm{Cmol}^{(+)} \mathrm{kg}-1$, lo cual puede aumentar los valores 
de $\mathrm{pH}$ por arriba de 8 (Khadka et al., 2017). De la misma manera, el Mg aumenta su biodisponibilidad en valores de $\mathrm{pH}$ alcalinos, por encima de 8.4 en la escala de $\mathrm{pH}$ se registran alrededor de $3.5 \mathrm{Cmol}^{(+)} \mathrm{kg}-1$ de $\mathrm{Mg}$ (Francos et al., 2018).

Tabla 1. Resultados de las propiedades físicas y químicas del suelo

\begin{tabular}{|c|c|c|c|}
\hline \multicolumn{2}{|c|}{ Propiedades físicas } & \multicolumn{2}{|c|}{ Propiedades químicas } \\
\hline Parámetro & Resultado & Parámetro & Resultado \\
\hline \multirow[t]{3}{*}{$\%$ Arena } & \multirow[t]{3}{*}{67} & $\mathrm{pH}$ & 8.2 \\
\hline & & CE & $8.5 \mathrm{dS} / \mathrm{m}$ \\
\hline & & Materia orgánica & $2.5 \%$ \\
\hline \multirow[t]{3}{*}{$\%$ Arcilla } & \multirow[t]{3}{*}{17} & Nitrógeno & $0.56 \%$ \\
\hline & & Calcio & $9.28 \mathrm{Cmol}^{(+)} \mathrm{kg}^{-1}$ \\
\hline & & Magnesio & $3.09 \mathrm{Cmol}^{(+)} \mathrm{kg}^{-1}$ \\
\hline \multirow[t]{3}{*}{ \%6 Limo } & \multirow[t]{3}{*}{16} & Cloruros & $125.35 \mathrm{Cmol}^{(+)} \mathrm{kg}^{-1}$ \\
\hline & & Cobre & $2.225 \mathrm{ppm}$ \\
\hline & & Hierro & $2.7 \mathrm{ppm}$ \\
\hline \multirow[t]{3}{*}{ Humedad } & \multirow[t]{3}{*}{$14.35 \mathrm{~g} / 100 \mathrm{~g}$} & Manganeso & $6.97 \mathrm{ppm}$ \\
\hline & & Fósforo & $2.24 \mathrm{Cmol}^{(+)} \mathrm{kg}^{-1}$ \\
\hline & & Potasio & $7.4 \mathrm{Cmol}(+) \mathrm{kg}^{-1}$ \\
\hline \multirow[t]{4}{*}{ Densidad real } & \multirow[t]{4}{*}{$1.3 \mathrm{~g} / \mathrm{cm}^{3}$} & Azufre & $0.73 \mathrm{Cmol}^{(+)} \mathrm{kg}^{-1}$ \\
\hline & & Zinc & $0.65 \mathrm{ppm}$ \\
\hline & & Sodio & $2750 \mathrm{ppm}$ \\
\hline & & PSI & $19.89 \%$ \\
\hline
\end{tabular}

Fuente: Elaboración propia. Delgado-González (2019)

\section{COMENTARIOS FINALES}

\subsection{CONCLUSIONES}

Los resultados evalúan al suelo con problemas de salinidad y baja disponibilidad de algunos nutrientes principalmente por los valores de $\mathrm{pH}$ y CE. Es indispensable considerar que la caracterización de suelos es un paso inicial hacia estudios de profundidad en relación a la zona de rizósfera para recuperación de la fertilidad del suelo. La importancia de esta investigación hace hincapié en la relación de la biodisponibilidad de micronutrientes y algunos macronutrientes, basados en las propiedades físicas y químicas del suelo como $\mathrm{pH}, \mathrm{CE}$, textura, entre otras más.

\subsection{RECOMENDACIONES}

Para futuras investigaciones relacionadas a las características fisicoquímicas en suelos salinos es necesario considerar la época del año en la que se realice la colecta, así como realizar una evaluación de la microbiota en la zona de la rizosfera y con ello proponer un modelo biológico de recuperación de sitios agrícolas perturbados por sales solubles. Estos análisis forman parte integral de investigaciones relacionadas al suelo, ya que dan robustez a los resultados y pueden establecer relaciones entre distintos factores asociados a la salinidad. 


\section{REFERENCIAS}

Adnan, M., Shah, Z., Fahad, S., Arif, M., Alam,M., Khan, I. A., Mian, I. A., Basir, A., Ullah, H., Arshad, M., Rahman, I. U., Saud, S., Ihsan, M., Jamal, Y., Amanullah, H., Hammad, H. M. y Nasim, W. (2017) Phosphate-Solubilizing Bacteria Nullify the Antagonistic Effect of Soil Calcification on Bioavailability of Phosphorus in Alkaline Soils. Scientific Reports. 7: 1 - 15.

Ahmad, P., Sharma, S. (2008). Salt stress and phyto-biochemical responses of plants. Plant Soil Environ. 54 (3), 89-99.

Aslam R., Bostan N., Nabgha-e-Amen, M. M. y Safdar W. 2011. A critical review on halophytes: salt tolerant plants. Journal of Medicinal Plants Research. 5(33): 7108-7118.

Behera, S. K. y Shukla, A. K. (2014) Spatial Distribution of Surface Soil Acidity, Electrical Conductivity, Soil Organic Carbon Content and Exchangeable Potassium, Calcium and Magnesium in Some Cropped Acid Soils of India. Land Degradation \& Development. 50 (1): 1 - 15.

Bravo, S., Amorós, J. A., Pérez-de-los-Reyesa, C., García, f. J., Moreno, M. M., Sánchez-Ormeño, M y Higueras, P. (2017) Influence of the soil $\mathrm{pH}$ in the uptake and bioaccumulation of heavy metals (Fe, $\mathrm{Zn}$, $\mathrm{Cu}, \mathrm{Pb}$ and $\mathrm{Mn}$ ) and other elements $(\mathrm{Ca}, \mathrm{K}, \mathrm{Al}, \mathrm{Sr}$ and $\mathrm{Ba}$ ) in vine leaves, Castilla-La Mancha (Spain). Journal of Geochemical Exploration. 174: 79 - 83.

Brooker, A. P., Lindsey, L. E., Culman, S. W., Subburayalu, S. K. y Thomison, P. R. (2017) Low Soil Phosphorus and Potassium Limit Soybean Grain Yield in Ohio. Cftm. 3 (1): 1 - 5.

Cao, J., Li, X., Kong, X., Zed, R., Dong, L., 2012. Using alfalfa (Medicago sativa) to ameliorate saltaffected soils in Yingda irrigation district in Northwest China. Acta Ecol. Sinica 32 (2), 68-73

Chhabra, R. 2017. Soil Salinity and Water Quality. Editorial Taylor and Francis. Estados Unidos de América. $\quad 16-24$.

De Sutter, T., Franzen, D., He, Y., Wick, A., Lee, J., Deutsch, B. y Clay, D. (2015) Relating Sodium Percentage to Sodium Adsorption Ratio and its Utility in the Northern Great Plains. Soil Science Society of America Journal Abstract - Nutrient Management \& Soil \& Plant Analysis Note. 79 (4): 1261 - 1264.

Duplay, J., Semhi, K., Errais, E., Imfeld, G., Babcsanyi, I. y Perrone, T. (2014) Copper, zinc, lead and cadmium bioavailability and retention in vineyard soils (Rouffach, France): The impact of cultural practices. Geoderma. 230: 318 - 328 .

Etxeberri, J.M. y J.A. Blanco Gorrichóa. "Un método óptimo para la extracción de proteínas del mero en Bilbao," Revista Castellana (en línea), Vol. 2, No. 12, 2003, consultada por Internet el 21 de abril del 2004. Dirección de internet: http://revistacastellana.com.es.

Francos, M., Pereira, P., Alcañiz, M. y Úbeda, X. (2018) Post-wildfire management effects on short-term evolution of soil properties (Catalonia, Spain, SW-Europe). Science of the Total Enviroment. 633: 285 292.

Gupta, S., \& Goyal, M. (2017). Soil salinity management in agriculture technological advances and applications (Innovations in Agricultural and Biological Engineering). Waretown, NJ: Apple Academic Press. 
Isbell, R. F. 2016. The Australian Soil Classification. Segunda edición. Australia. 43 - 45.

Hazourli, A., Hazourli, S., Nacer, H. y Fernandez, M. A. (2016) Feasibility of electrokinetic nitrogen supply for soil fertilization purposes. J. Mater. Environ. Sci. 7 (4): 1072 - 1079.

Huang, J., Davies, G. B., Bowd, D., Monteiro-Santos, F. A. y Triantafilis, J. (2014) Spatial prediction of the exchangeable sodium percentage at multiple depths using electromagnetic inversion modelling. Soil Use and Management. 30 (2): 241 - 250.

Khadka, D., Lamichhane, S., Tiwari, D. N. y Mishra, K. (2017) Assessment of soil fertility status of national rice research program, Hardinath, Dhanusha, Nepal. Scientific Research Council. 3 (1): 86 - 105.

Katerji, N., van Hoorn, J.W., Hamdy, A., Mastrorilli, M., 2000. Salt tolerance classification of crops according to soil salinity and to water stress day index. Agric. Water Manage. 43 (1), 99-109.

Martínez-González, F., Sosa-Pérez, F. y Ortiz-Medel, J. (2010) T Comportamiento de la humedad del suelo con diferente cobertura vegetal en la Cuenca La Esperanza. Tecnología y ciencias del agua. 1 (4): $1-15$.

Mehlich A. (1984). Mehlich 3 soil test extractant: A modification of Mehlich 2 extractant. Comm. Soil Sci. Plant An., (15) 1409- 1416.

Nouri, H., Chavoshi Borujeni, S., Nirola, R., Hassanli, A., Beecham, S., \& Alaghmand, S. et al. (2017). Application of green remediation on soil salinity treatment: A review on halophytoremediation. Process Safety and Environmental Protection, (107), 94-107. Retrieved from https://doi.org/10.1016/j.psep.2017.01.021.

Qadir, M., Ghafoor A. y Murtaza, G. 2000. Amelioration strategies for saline soils: a review. Land Degradation and Development. 11: 501 - 521.

$\mathrm{S}$

Read, D. S., Matzke, M., Gweon, H. S., Newbold, L. K., Heggelund, L., Diez-Ortiz, M., Lahive, E., Spurgeon, D. y Svendsen. C. (2016) Soil pH effects on the interactions between dissolved zinc, non-nanoand nano-ZnO with soil bacterial communities. Environmental Science and Pollution Research. 23 (5): $4120-4128$.

Redžić, S., Sijarić, G., Muhić-Šarac, T., Pehić, E. y Hrnjica, D. (2014) Distribution and bioavailability of manganese in soil in the vicinity of the "Bužim" abandoned mine. Geologia Croatica. 67 (1): 45 - 58.

Sameen R., I., Syed W., S., \& Alvina G., K. (2016). Phytoremediation of Saline Soils for Sustainable Agricultural Productivity. In A. Parvaiz, Plant Metal Interaction Emerging Remediation Techniques (1st ed., pp. 465-468). Oxford, UK: Elsevier Inc. Retrieved from https://doi.org/10.1016/B978-0-12-8031582.00018-7

Sarwar, N., Imran, M., Shaheen, M.R., Ishaque, W., Kamran, M.A., Matloob, A., Rehim, A. y Hussain, S. (2017). Phytoremediation strategies for soils contaminated with heavy metals: modifications and future perspectives. Chemosphere 102: $710-721$.

Srivastava N. (2020) Reclamation of Saline and Sodic Soil Through Phytoremediation. In: Shukla V., Kumar N. (eds) Environmental Concerns and Sustainable Development. Springer, Singapore. https://doi.org/10.1007/978-981-13-6358-0_11 
Vasques, G. M., Demattê, J. A. M.,Viscarra-Rossel, R. A., Ramírez-López, L. y Terra, F. S. (2014) Soil classification using visible/near-infrared diffuse reflectance spectra from multiple depths. Geoderma. 223: $73-78$.

Wong, V.N.L., Greene, R.S.B., Dalal, R.C., Murphy, B.W., 2010. Soil carbon dynamics in saline and sodic soils: a review. Soil Use Manage. 26 (1), 2-11.

Xua, D. C., Zhong, C., Yina, K., Peng, S., Zhu, T. y Cheng, G. (2018) Alkaline solubilization of excess mixed sludge and the recovery of released phosphorus as magnesium ammonium phosphate. Bioresource Technology. 249: $783-790$.

Zhalnina, K., Dias, R., Dörr de Quadros, P., Davis-Richardson, A., Camargo, F. A. O., Clark, I. M., McGrath, S. P., Hirsch, P. R. y Triplet, E. W. (2015) Soil pH Determines Microbial Diversity and Composition in the Park Grass Experiment. Microbial Ecology. 69 (2): 395 - 406.

\section{NOTAS BIOGRÁFICAS}

El M. en C. Cristián Raziel Delgado-González estudió la Licenciatura en Biología y la Maestría en Ciencias en Biodiversidad y Conservación. Actualmente es estudiante del Doctorado en Ciencias Agropecuarias en el Instituto de Ciencias Agropecuarias, el cual pertenece a la Universidad Autónoma del Estado de Hidalgo. Su área de enfoque es la morfofisiología y la ecofisiología vegetal, así como el enfoque hacia la biorremediación de suelo.

El Dr. Rodrigo Rodríguez-Laguna es especialista en manejo de recursos forestales. Ha dirigido diversas tesis de Licenciatura, Maestría y doctorado, así como colaborado en proyectos nacionales y estatales relacionados a especies forestales en proyectos CONACYT y CONAFOR. Actualmente es profesor investigador de tiempo completo en la Universidad Autónoma del Estado de Hidalgo y cuenta con publicaciones relacionadas a recursos forestales.

El Dr. Juan Capulín-Grande es especialista en edafología con enfoque a la nutrición vegetal. Fue coordinador de invernaderos a nivel estatal, así como profesor investigador titular en el área académica de ciencias agrícolas y forestales del Instituto de Ciencias agropecuarias de la UAEH. Ha colaborado en proyectos CONACYT y CONAFOR. Actualmente es profesor investigador titular de la Universidad Autónoma del estado de Hidalgo.

El Dr. Alfredo Madariaga-Navarrete es especialista en el al área de biotecnología y Ciencias Agropecuarias, enfocado al control biológico en la producción agrícola sustentable. Ha colaborado en proyectos SAGARPA- CONACYT y es consultor en la iniciativa privada. Ha dirigido tesis a nivel de licenciatura y posgrado. Es profesor investigador titular en el área académica de ciencias agrícolas y forestales del Instituto de Ciencias agropecuarias de la UAEH.

La Dra. Margarita Islas-Pelcastre es especialista Ciencias Ambientales enfocada a la recuperación y aprovechamiento sustentables de sitios contaminados. Responsable técnico en proyectos financiados FESE 2014, SAGARPA 2016 y SAGARPA-CONACYT 2018. Cuenta con un registro de patente No. $\mathrm{Mx} / \mathrm{a} / 2017 / 016042$. Ha dirigido tesis a nivel de licenciatura y posgrado. Es profesor investigador en el área académica de Ingeniería Agroindustrial y Alimentos en el Instituto de Ciencias agropecuarias de la UAEH. 